This is the author's final, peer-reviewed manuscript as accepted for publication. The publisher-formatted version may be available through the publisher's web site or your institution's library.

\title{
Individual differences in impulsive and risky choice: effects of environmental rearing conditions
}

Kimberly Kirkpatrick, Andrew T. Marshall, Aaron P. Smith, Juraj Koci, and Yoonseong Park

\section{How to cite this manuscript}

If you make reference to this version of the manuscript, use the following information:

Kirkpatrick, K., Marshall, A. T., Smith, A. P., Koci, J., \& Park, Y. (2014). Individual differences in impulsive and risky choice: Effects of environmental rearing conditions. Retrieved from http://krex.ksu.edu

\section{Published Version Information}

Citation: Kirkpatrick, K., Marshall, A. T., Smith, A. P., Koci, J., \& Park, Y. (2014). Individual differences in impulsive and risky choice: Effects of environmental rearing conditions. Behavioural Brain Research, 269, 115-127.

Copyright: @ 2014 Elsevier B.V.

Digital Object Identifier (DOI): doi:10.1016/j.bbr.2014.04.024

Publisher's Link: http://www.sciencedirect.com/science/article/pii/S0166432814002423

This item was retrieved from the K-State Research Exchange (K-REx), the institutional repository of Kansas State University. K-REx is available at http://krex.ksu.edu 
Individual differences in impulsive and risky choice: Effects of environmental rearing conditions

$$
\begin{aligned}
& \text { Kimberly Kirkpatrick }{ }^{1} \text {, Andrew T. Marshall }{ }^{1} \text {, Aaron P. Smith }{ }^{3} \text {, } \\
& \text { Juraj Koci }{ }^{2} \text {, and Yoonseong Park }{ }^{2} \\
& { }^{1} \text { Department of Psychological Sciences, Kansas State University } \\
& { }^{2} \text { Department of Entomology, Kansas State University } \\
& { }^{3} \text { Department of Psychology, University of Kentucky }
\end{aligned}
$$

Corresponding author: Kimberly Kirkpatrick, Department of Psychological Sciences, 492 Bluemont Hall, 1100 Mid-Campus Drive, Kansas State University, Manhattan, KS 665065302. E-mail: kirkpatr@ksu.edu Tel: 785-532-0805. Fax: 785-532-5401

Running Head: Individual differences in impulsive and risky choice 


\begin{abstract}
The present experiment investigated early-rearing environment modulation of individual differences in impulsive and risky choice. Rats were reared in an isolated condition (IC; $n=12$ ), in which they lived alone without novel stimuli, or an enriched condition $(\mathrm{EC} ; n=12)$, in which they lived among conspecifics with novel stimuli. The impulsive choice task involved choices between smaller-sooner (SS) versus larger-later (LL) rewards. The risky choice task involved choices between certain-smaller (C-S) versus uncertain-larger (U-L) rewards. Following choice testing, incentive motivation to work for food was measured using a progressive ratio task and correlated with choice behavior. HPLC analyses were conducted to determine how monoamine concentrations within the prefrontal cortex (PFC) and nucleus accumbens (NAC) related to behavior in different tasks. IC rats were more impulsive than EC rats, but they did not differ in risky choice behavior. However, choice behavior across tasks was significantly correlated (i.e., the more impulsive rats were also riskier). There were no group differences in monoamine levels, but noradrenergic and serotonergic concentrations were significantly correlated with impulsive and risky choice. Furthermore, serotonin and norepinephrine concentrations in the NAC significantly correlated with incentive motivation and the timing of the reward delays within the choice tasks. These results suggest a role for domain general processes in impulsive and risky choice and indicate the importance of the NAC and/or PFC in timing, reward processing, and choice behavior.

Keywords: impulsive choice; risky choice; differential rearing; individual difference; monoamine concentration; rat
\end{abstract}




\section{Introduction}

Individual differences in impulsive and risky behaviors have been identified as predictors of substance abuse and pathological gambling (e.g., Bickel \& Marsch, 2001; Carroll, Anker, \& Perry, 2009; de Wit, 2008; Perry \& Carroll, 2008). Impulsive and risky choice have also been identified as potential trait variables, showing test-rest reliability statistics in the range of other personality variables in human participants (Jimura et al., 2011; Kirby, 2009; Matusiewicz, Carter, Landes, \& Yi, 2013; Odum, 2011a, 2011b; Odum \& Baumann, 2010; Ohmura, Takahashi, Kitamura, \& Wehr, 2006; Peters \& Büchel, 2009). In addition, stable individual differences in impulsive choice have been reported in rats across different choice parameters, suggesting that impulsive choice may also serve as a trait variable in rats (Galtress, Garcia, \& Kirkpatrick, 2012; Garcia \& Kirkpatrick, 2013; Marshall, Smith, \& Kirkpatrick, under review). Impulsive choice is often defined as the choice for smaller-sooner (SS) over larger-later (LL) rewards (e.g., Ainslie, 1975), while risky choice is often defined as the choice for uncertainlarger (U-L) over certain-smaller (C-S; e.g., Kahneman \& Tversky, 1979) rewards. The fact that both impulsive and risky choice behaviors are risk factors in similar problem behaviors suggests the possibility of shared underlying processes. In addition, both impulsive and risky choice can be accounted for by the hyperbolic discounting equation, $V=A /(1+k X)$, where $V$ is the subjective value of reward and $A$ is the amount of reward (Mazur, 1987, 2001; Rachlin, Raineri, \& Cross, 1991). In impulsive choice, $X$ is the delay to reward, which decreases the value of a reward as delay increases, thus leading to the discounting of reward value with delay. In risky choice, $X$ is the odds against receiving the larger risky reward, which leads to the discounting of probabilistic rewards. In both cases, $k$ is the discounting rate which determines the effect of delay or probability on subjective value and serves as an individual difference parameter. 
Despite the similar patterns of impulsive and risky choice, and their shared correlation with other behaviors, there is surprisingly little research examining their interrelationship. The few examinations that have been undertaken have revealed inconsistent results, with weak to moderate correlation patterns in individual differences in impulsive and risky choice behavior in humans (Baumann \& Odum, 2012; Myerson, Green, Hanson, Holt, \& Estle, 2003; Peters \& Büchel, 2009; Richards, Zhang, Mitchell, \& de Wit, 1999), and moderately strong correlations in pigeons (Laude, Beckman, Daniels, \& Zentall, 2014).

Neurobiological studies have indicated partial overlap in the substrates involved in impulsive and risky choice and their component processes of delay, probability, and reward processing (see Doya, 2008; Peters \& Büchel, 2011; Platt \& Huettel, 2008). The nucleus accumbens (NAC) and prefrontal cortex (PFC) are involved in both impulsive and risky choice (Ballard \& Knutson, 2009; Cardinal \& Howes, 2005; Doya, 2008; Galtress \& Kirkpatrick, 2010; Mobini et al., 2002; Peters \& Büchel, 2011; Platt \& Huettel, 2008; Stopper \& Floresco, 2011), as are dopamine (DA) and serotonin (5-HT) levels (Brunner, 1997; Campbell-Meiklejohn et al., 2011; Cocker, Dinelle, Kornelson, Sossi, \& Winstanley, 2012; Evenden, 1999; Winstanley, Theobald, Dalley, Cardinal, \& Robbins, 2006; Zhong et al., 2009). Therefore, given the shared neural mechanisms of impulsive and risky choice, one would expect to observe behavioral correlations as well.

Peters and Büchel (2009) proposed that domain general and specific processes contribute to impulsive and risky choice. Domain-general reward processes emerge from the NAC and PFC, and involve shared processes related to the overall valuation and incentive salience of rewards. Domain-general processes should result in comorbidities of impulsive and risky choice. On the other hand, domain-specific processes are involved in the determination of task-specific 
information. In impulsive choice, delay is a task-specific factor, whereas probability is specific to risky choice. Magnitude is involved in both of these tasks and would produce some shared task variance (although magnitude would still be considered domain specific as it would not contribute to other choice tasks such as effort discounting or social discounting). Given that domain-general processes participate in both tasks, coupled with the involvement of reward magnitude processing in both impulsive and risky choice, one would expect to observe robust correlations in individual differences in impulsive and risky choice. Accordingly, the present study sought to further assess the presence of these correlations in individual rats.

One variable that has been shown to participate in impulsive and risky choice, dopaminergic and serotonergic function, and neural functioning within the NAC and PFC is the post-weaning rearing environment (Brenes, Rodriguez, \& Fornaguera, 2008; Hellemans, Nobrega, \& Olmstead, 2005; Kirkpatrick, Marshall, Clarke, \& Cain, 2013; Perry, Stairs, \& Bardo, 2008; Rahman \& Bardo, 2008; Wood, Buse, Wellman, \& Rebec, 2005; Wood \& Rebec, 2009; Zeeb, Wong, \& Winstanley, 2013; Zhu, Apparsundarum, Bardo, \& Dwoskin, 2005). The differential rearing paradigm has typically compared rats raised in an isolated condition (IC) and an enriched condition (EC; Renner \& Rosenzweig, 1987; Simpson \& Kelly, 2011). EC rats are housed with other rats and novel objects, whereas IC rats are housed individually without novel objects. The present study sought to determine whether these extreme rearing conditions would interact with individual differences in impulsive and risky choice. If rearing conditions moderate individual differences, then this could provide a potential route for the development of future interventions to decrease impulsive and risky choice behaviors, as well as providing some further understanding of mechanisms of impulsive and risky choice. 
Accordingly, the goal of the present report was to investigate the moderation of individual differences in impulsive and risky choice by differential rearing, examining both the relevant behavioral and neurobiological processes. Despite the effects of differential rearing on brain and behavioral functioning, there has not been, to our knowledge, a comprehensive analysis of an interaction of rearing environment with individual differences. In addition, relatively little is known about the effects of rearing environment on comorbidities of impulsive and risky choice. Therefore, the present study sought to yield further understanding of these processes. We also included measures of timing and reward processing as potential indices of domain-specific processes, and their possible modulation by environmental rearing, relationship with impulsive and risky choice, and correlations with monoamine concentrations.

\section{Materials and Methods}

\subsection{Animals}

Twenty-four experimentally naïve male Sprague-Dawley rats arrived to the colony at Kansas State University (Manhattan, KS) at 21 days of age (Charles River, Portage, MI). Their housing conditions varied according to their group assignment (see 2.3.1. Environmental rearing). Following rearing, and prior to the onset of behavioral testing, the rats were placed on a restricted diet to achieve $85 \%$ of their target weights determined by growth charts obtained from the supplier. During behavioral testing, the rats received part of their daily ration in the form of 45-mg pellets (BioServ, Frenchtown, NJ) that were delivered in the experimental chambers. The rats had free access to water at all times. The colony room was maintained on a 12:12 $\mathrm{hr}$ reversed light:dark cycle with the lights off at $8 \mathrm{a} . \mathrm{m}$. Several red lamps illuminated the room during the dark cycle.

\subsection{Apparatus}


2.2.1. Locomotor testing. Environmental rearing is known to produce robust and longlasting effects on locomotor behavior (e.g., Simpson \& Kelly, 2011), so this assay assessed the effectiveness of the rearing conditions. Locomotor testing was conducted in an enclosed, novel environment measuring $40.6 \times 40.6 \times 40.6 \mathrm{~cm}$. The chamber consisted of Plexiglas walls and plastic flooring that was covered with bedding. The chamber also had a photo beam sensor ring that consisted of a $16 \times 16(\mathrm{x}$-axis) photocell array that created a detection grid with individual beams spaced $2.5 \mathrm{~cm}$ apart (TruScan 2.01, Coulbourn Instruments, Whitehall, PA) and was linked to a personal computer. Photobeam interruptions were recorded every $50 \mathrm{~ms}$. A whitenoise generator $(\sim 70 \mathrm{~dB})$ was used to mask extraneous sounds.

2.2.2. Operant behavioral testing. The choice and progressive ratio procedures were conducted in 24 identical operant chambers (Med Associates, St. Albans, VT). Each chamber measured $25 \times 30 \times 30 \mathrm{~cm}$ and was housed inside a ventilated, noise attenuating box measuring $74 \times 38 \times 60 \mathrm{~cm}$. The chambers were located in two separate rooms, with 12 chambers in each room. Two levers (ENV-122CM) were situated on either side of the food cup and presses were recorded by a micro-switch. Nose poke keys with cue lights (ENV-119M-1) were located directly above each lever. A magazine pellet dispenser (ENV-203) delivered 45-mg food pellets into the food cup. Water was available through a metal tube located on the lower-center of the back wall, directly opposite the food cup. MED-PC IV controlled the experiment and recorded the time of events with a 2-ms resolution (Tatham \& Zurn, 1989).

\subsection{Procedure}

2.3.1. Environmental rearing. Upon arrival, the rats were pair-housed in standard shoebox cages for two days prior to the initial activity test. They were then randomly assigned to initial groups and their activity levels in the locomotor chamber were assessed for any 
differences. The groups did not differ significantly in their pre-rearing activity levels $(p>.05)$, so the initial random assignment was maintained. The two groups were differentially reared in either an enriched (EC) or impoverished (IC) condition $(n=12)$ for the duration of the study. All EC rats were housed together in a single large metal cage $(60 \times 120 \times 45 \mathrm{~cm})$ with pulp paper bedding. They were given 14 novel objects (i.e. PVC pipe, buckets, children's toys, etc.), seven of which were replaced daily with seven new objects. Once per week, all objects in the cage were replaced with new items. The IC rats were housed individually in hanging wire cages with a wire mesh floor and front panel $(17 \times 24 \times 20 \mathrm{~cm})$, and solid metal sides, back, and top. IC rats were not handled during the rearing period, while EC rats were handled daily. The EC and IC conditions represent the extremes of the animal husbandry spectrum, and these extremes were employed to maximize the potential moderation of individual differences across the spectrum of rearing conditions.

2.3.2. Locomotor activity. The rats were placed in locomotor chambers for $60 \mathrm{~min}$ on the second day after arrival (post-natal day, PND 23) and again following the rearing period (PND 55). During the first screen the chamber floors were covered with pine chip bedding and Plexiglas walls, while the second screen used corn cob bedding and the walls were covered with opaque plastic sheets to maintain the novelty of the locomotor chambers.

2.3.3. Pre-training. Prior to the onset of operant behavioral testing (starting on PND 69), the rats were trained to eat from the food receptacle on an random time $60 \mathrm{~s}$ schedule (1 session) followed by training to press the left and right levers. Lever press training (1 session) consisted of blocks of an incrementing response requirement: fixed ratio 1, random ratio 3 , and random ratio 5. There were four sub-blocks within each block in which the rat was exposed to both levers until 5 rewards were earned on each lever. We have previously used this procedure 
(e.g., Garcia \& Kirkpatrick, 2013) as it results in steady rates of lever pressing and minimizes initial lever biases. We did not observe any significant differences in initial lever preferences between the two rearing conditions $(p>.05)$.

2.3.4. Impulsive choice task. The rats received an impulsive choice task with magnitudes that varied across phases (see Garcia \& Kirkpatrick, 2013; Marshall et al., under review). In Phase 1, the rats were given a choice between a 10-s, 1-pellet SS option and a 30-s, 1-pellet LL option to establish choice behavior in the absence of differential rewards. Subsequently, in Phases 2 and 3, the LL reward increased to 2 and then 3 pellets. Each phase lasted for 10 sessions. The impulsive choice task consisted of an intermixture of free choice, forced choice, and peak trials in each session. There were 100 total trials delivered in two 50trial blocks. Each trial block consisted of 30 free choice, 8 SS forced choice, 8 LL forced choice, 2 SS peak, and 2 LL peak trials, randomly intermixed. A 60-s intertrial interval (ITI) followed each trial. On free choice trials, both levers were initially inserted. After one lever was pressed, the alternate lever retracted, the cue light above the lever was illuminated, and the FI schedule (10-s for the SS and 30-s for the LL) began. The first response following the delay criterion resulted in reward delivery, lever retraction, cue light offset, and delivery of the ITI. Forced choice trials began with the insertion of the SS or LL lever, and the first lever press initiated the FI duration (10 or $30 \mathrm{~s})$; these trials were otherwise the same as free choice trials. Peak trials (Roberts, 1981) also began with the insertion of either the SS or the LL lever, with a lever press initiating the trial, which lasted for $90 \mathrm{~s}$. No food was delivered on peak trials, and lever presses were recorded to assess timing of the SS and LL delays. Sessions lasted approximately $2.5 \mathrm{hr}$, and training continued for 10 sessions in each phase. Half of the rats in each condition received the impulsive choice task first and half received the risky choice task first. 
2.3.5. Risky choice task. The risky choice task consisted of variations of the probability of reward for U-L choices (Marshall \& Kirkpatrick, 2013). The risky choice task was identical to the impulsive choice task except for the nature of the two outcomes. The C-S option always delivered a reward magnitude of either 1 or 3 pellets $(p=.5)$. The U-L choices probabilistically delivered either 3 or 9 pellets, with probability of U-L reward varying across phases that lasted for 10 sessions each: $.17, .33, .5$, and .67 . For example, with a probability of .5 , the rats would receive 0,3 , or 9 pellets with the probabilities of $.5, .25$, and .25 , respectively. The delay to reward was $20 \mathrm{~s}$ for both options and the ITI was $60 \mathrm{~s}$.

2.3.6. Progressive ratio task. Rats were given a progressive ratio (PR) 3 task to assess their motivation to work for rewards of different magnitudes and probabilities. Food was delivered after 3 lever presses on the first trial, and the response requirement increased by 3 presses on each subsequent trial. The PR incremented until the rat stopped pressing for $10 \mathrm{~min}$, at which time a 20-min inter-block interval (IBI) ensued, and the required number of lever presses was reset to 3 for next block. Rats were tested for two blocks and the last PR completed in each block was recorded as the breakpoint. The rats received 2 sessions of each of the magnitude/probability combinations: $1 / 100,3 / 100,3 / 67$, and 3/17. The PR was given on the left lever, which was the SS/C-S lever for half of the rats and the LL/U-L lever for the other half. PR training ended on PND 164.

2.3.7. High performance liquid chromatography (HPLC). Following behavioral testing, the rats were euthanized and their brains removed for analysis of monoamine concentrations. The NAC and PFC were extracted and frozen at $-80^{\circ} \mathrm{C}$. For analysis of monoamines, each tissue sample was homogenized by a plastic pestle in $100 \mu \mathrm{l}$ of homogenization solution containing 0.2M perchloric acid (PCA), $100 \mu \mathrm{M}$ EDTA.2Na and $1 \mathrm{ng}$ 
of internal standard isoproterenol (ISO) per $20 \mu \mathrm{l}$ of solution. After homogenization, additional homogenization solution was added according to the milligram tissue weight to obtain a final 5fold volume (e.g., $100 \mathrm{mg}=500 \mu \mathrm{l})$. The tissue homogenate was incubated on ice bath for 30 min and centrifuged at $20000 \mathrm{~g}$ and $4^{\circ} \mathrm{C}$ for $15 \mathrm{~min}$. Supernatant was removed, filtrated in a 0.45 $\mu \mathrm{m}$ PVDF filter column (12000g for $4 \mathrm{~min})$ and analyzed or stored at $-80^{\circ} \mathrm{C}$ until analysis. HPLC analyses were performed using HTEC-500 (Eicom Corp., Japan), standalone HPLC system incorporating an electrochemical detector consisting of counter electrode, pure graphite WE-PG working electrode and Ag/AgCl RE-100 reference electrode. Separation HPLC column SC-50DS, $3 \mathrm{~mm} \times 150 \mathrm{~mm}$ (Eicom Corp., Japan), was with a pre-column, packed with AC-0DS packing matrix (Eicom Corp., Japan). Analyses of monoamines were carried out by injecting $20-\mu l$ injection volumes, consisting of $10 \mu \mathrm{l}$ of sample aliquot and $10 \mu \mathrm{l}$ of homogenization solution. The flow rate was $500 \mu 1 / \mathrm{min}$ with a mobile phase solution containing citric acid (8.4), sodium acetate (3.1), sodium 1-octanesulfonate (0.22), EDTA.2Na (0.05 g/L) and methanol (15\%), $\mathrm{pH} 3.5$. The effluent was monitored electrochemically at a potential of $+500 \mathrm{mV}$ versus $\mathrm{Ag} / \mathrm{AgCl}$.

\subsection{Data Analysis}

2.4.1. Locomotor activity. Locomotor activity was detected by an infrared-beam array that tracked the location of the rat in consecutive 0.5 -s intervals; the location of the rat was defined as the center of all beams crossed in the 0.5 -s interval. The total distance moved (m) was derived from the change in location across the test session. The locomotor testing results were subjected to a $2 \times 2$ repeated measures analysis of variance (ANOVA) with rearing condition (EC, IC) as the between-subjects variable and pre/post (pre-rearing, post-rearing) as the within subjects variable. 
2.4.2. Choice behavior. Analyses of risky and impulsive choice behavior were conducted on the last five sessions of each phase. Choice behavior was measured with an empirical $\log$ odds ratio, $\log$ odds $=\log \left(\mathrm{N}_{\mathrm{SS}}+.5 / \mathrm{N}_{\mathrm{LL}}+.5\right)$, in which $\log$ is the natural logarithm of the odds ratio, $\mathrm{N}_{\mathrm{SS}}$ is the total SS choices and $\mathrm{N}_{\mathrm{LL}}$ is the total LL choices. The addition of .5 to the numerator and denominator avoids computational problems associated with exclusive choice (Garcia \& Kirkpatrick, 2013; Haldane, 1956). Analyses of the log odds ratios were conducted using a $3 \times 2$ repeated measures ANOVA for impulsive choice and a $4 \times 2$ repeated measures ANOVA for risky choice with a within-subjects variable of LL magnitude (for impulsive choice) or U-L probability (for risky choice) and a between-groups variable of rearing condition (EC, IC).

Two additional metrics of choice behavior were determined for each rat to disentangle the possible effects of rearing condition on bias to select the more impulsive/risky choice versus adaptability in choice behavior. The mean of the log odds across phases within each task was used as a measure of overall bias and the slope of the function relating log odds to LL magnitude or U-L probability as a measure of adaptability to changes in magnitude or probability.

2.4.3. Peak trial timing behavior. Tracking of the delay to reward in the impulsive and risky choice tasks was measured on each peak trial using a low-high-low (LHL) algorithm (Church, Meck, \& Gibbon, 1994; Galtress \& Kirkpatrick, 2009). Response rates on peak trials follow the pattern of an initial low state of responding followed by a high state of responding that usually straddles the expected time of reward, and then followed by a second low state of responding. The LHL algorithm is used to identify the times of high states of responding on individual trials by determining the best-fitting square wave to the set of individual responses on each trial, defined by: $A=d_{L 1}\left(r-r_{L 1}\right)+d_{H}\left(r_{H}-r\right)+d_{L 2}\left(r-r_{L 2}\right)$, where $r$ refers to the mean 
response rate over the trial duration, $\mathrm{d}_{\mathrm{L} 1}, \mathrm{~d}_{\mathrm{H}}$, and $\mathrm{d}_{\mathrm{L} 2}$ refer to the durations of the first low state, high state, and second low state, respectively, and $\mathrm{r}_{\mathrm{L} 1}, \mathrm{r}_{\mathrm{H}}$, and $\mathrm{r}_{\mathrm{L} 2}$ refer to the response rate in those states. The first low state is the time from the start of the trial until the start time of the high state, and the second low state is the time from the end of the high state until the end of the trial. The high state occupies the time between the two low states and must begin and end with a response. From the fitted square wave, indices of response timing can be extracted. The start time is the time of the response that initiates the high state relative to trial onset, the end time is the time of the last response in the high state, the middle time is the mean of the start and end time, and the duration is the difference of the start and end times. Responses prior to the start or following the end time are considered as low state responses, and all responses in between the start and end are considered as high state responses. The analysis was conducted across the last 5 sessions of each phase to mirror the choice analyses, and then averaged across phases for each choice task to increase stability of measurements of timing behavior due to the small number of peak trials per session.

2.4.4. PR behavior. PR behavior was measured with a break point, which was the last PR value completed before the rat stopped responding for $10 \mathrm{~min}$. If only one breakpoint was completed in a session, then that was used as the breakpoint for the session, but if two breakpoints were completed then the mean breakpoint was used. The analysis was conducted on the last session of each reward magnitude/probability phase.

\subsubsection{HPLC monoamine concentrations. Quantifications of monoamines were} performed in PowerChrom software (eDAQ Pty Ltd., Australia) using standard curves and the peak area method. Isoproterenol served as an internal standard to compensate for recovery rate 
variations in the purification process. Final monoamine concentrations in the samples were expressed as ng/mg of tissue weight.

2.4.6. Statistical analyses. The statistical analyses were conducted in SPSS using the repeated measures ANOVA techniques in the general linear model and bivariate Pearson correlation techniques. Tukey post-hoc tests were used to identify the source of significant interactions. The criterion for reporting of significant results was $p<.05$ in all cases.

\section{Results}

\subsection{Locomotor activity}

Figure 1 displays the results of the locomotor activity tests conducted immediately before (Pre) and after (Post) the 30-day rearing period. There were considerable individual differences in locomotor activity prior to rearing, with the two groups showing similar activity levels. Following rearing, all 12 of the IC rats increased their activity in the post-rearing test while the EC rats as a group maintained their activity levels, with some individuals showing decreases, and some showing increases. These general patterns were verified by an ANOVA, which revealed a significant Pre/Post $\times$ Rearing Condition interaction, $F(1,21)=21.4, p<.001, \eta_{\mathrm{p}}{ }^{2}=.51$, and significant main effects of pre/post, $F(1,21)=21.8, p<.001, \eta_{\mathrm{p}}{ }^{2}=.51$, and rearing condition, $F(1,21)=7.1, p=.014, \eta_{\mathrm{p}}{ }^{2}=.25$. The interaction was due to significantly greater activity in the IC rats (versus the EC rats) in the post-rearing test $(p<.05)$, whereas there were no group differences pre-rearing.

\subsection{Choice behavior}

3.2.1. Impulsive choice. Figure $2 \mathrm{~A}$ displays the log odds of impulsive (SS) choices as a function of LL magnitude. Both groups were sensitive to the change in LL magnitude, but the EC rats altered their choice behavior to a greater degree. This was verified by an ANOVA, 
which revealed a significant main effect of phase, $F(2,42)=70.5, p<.001, \eta_{\mathrm{p}}{ }^{2}=.77$, and a Phase $\times$ Rearing condition interaction, $F(2,42)=4.8, p=.013, \eta_{\mathrm{p}}{ }^{2}=.19$.

In addition to analyzing the choice data, the mean and slope of the log odds function was calculated (Section 2.4.2) to determine the effect of rearing condition on bias and adaptability, respectively. The mean versus slope is displayed in Figure 2B for the individual EC and IC rats. In general, low slopes indicate poor adaptation of choice behavior when the magnitude changed and high means indicate a bias towards impulsivity. Thus, rats in the lower-right quadrant of the decision space are SS responders (Garcia \& Kirkpatrick, 2013; Marco et al., 2009) that have an impulsive bias coupled with poor adaptability, whereas rats in the lower-left quadrant are LL responders with a flat slope and an LL bias, and rats near the top of the distribution are adaptive decision makers with more neutral biases. Figure 2B shows substantial individual differences in choice behavior, and that the IC rats were shifted towards the SS responder quadrant relative to the EC rats, which is reflected in their generally flatter choice functions (Figure 2A). Both the mean and slope were subjected to a one-way ANOVA to determine the effect of rearing condition on bias versus adaptability. This revealed a significant rearing condition effect on the slope of the impulsive choice function, $F(1,21)=6.3, p=.020, \eta_{\mathrm{p}}{ }^{2}=.23$, but no significant effect on the mean.

3.2.2 Risky choice. Figure $2 \mathrm{C}$ displays the choice results from the risky choice task as a function of U-L probability. Both groups were sensitive to the probability of food on the uncertain side. Although there was a tendency for the IC rats to choose the risky (U-L) option more often, this was not significant, but there was an overall effect of phase, $F(3,63)=51.3, p<$ $.001, \eta_{\mathrm{p}}^{2}=.71$. 
Figure 2D displays the risky choice mean versus slope for the individual EC and IC rats. Here, the lower-left quadrant contains individuals with a risk-averse bias and individuals in the lower-right are risk-prone. The majority of rats were adaptive decision makers with a steeper slope and fairly neutral bias. There was no obvious shift in the distribution of animals, but the IC rats did have a tighter distribution, and they were less likely to show a risk-averse bias than the EC rats. An ANOVA on the mean and slope of the risky function confirmed that there were no significant effects of rearing condition.

3.2.3. Inter-task correlations: Impulsive and risky choice. Given the considerable individual differences observed in both rearing conditions and in both tasks, a further analysis was conducted to examine inter-task correlations between means and slopes for the impulsive and risky choice tasks. The correlations are displayed in Figure 3 for the EC and IC rats. There was a significant positive correlation between the mean of the two tasks $(r=.83, p<.001$; Figure $3 \mathrm{~A})$, and the slopes were also positively correlated $(r=.68, p<.001$; Figure 3B).

\subsection{Peak trial timing behavior}

The four measures of timing behavior [start time (S), middle time (M), end time (E) and duration (D)] from the LHL analysis are displayed in Figure 4 for the individual EC and IC rats. Separate analyses are provided for the SS and LL levers in the impulsive choice task (Figure 4A and 4B) and for the C-S and U-L levers in the risky choice task (Figure 4C and 4D). In general, the rats in both groups accurately tracked the durations in both tasks. Middle times measuring the peak time of responding were close to the target durations ( $10 \mathrm{~s}$ for SS, $30 \mathrm{~s}$ for LL, and $20 \mathrm{~s}$ for C-S and U-L levers). The high states generally started at around $50 \%$ of the target duration and ended at around 2 times the target duration. There were, however, no effects of differential 
rearing on any of the metrics of timing, as assessed by one-way ANOVAs conducted separately for each measure on each lever.

\subsection{PR behavior}

The PR breakpoint for each of the reward magnitude and probability combinations is displayed in Figure 5. The IC rats displayed significantly longer breakpoints for the PR 1/100 condition, $F(1,21)=7.7, p=.011, \eta_{\mathrm{p}}{ }^{2}=.27$, but there were no effects of rearing condition on the other reward conditions.

\subsection{Inter-task correlations: Locomotor activity, impulsive and risky choice, timing behavior, and PR breakpoints}

To determine whether the performance on the locomotor activity and PR tasks was related to choice behavior at the individual rat level, cross-task correlations were conducted comparing post-rearing locomotor activity and the PR break point in each reward phase with the mean and slope of the impulsive and risky choice functions. Post-rearing locomotor activity did not correlate with impulsive choice, risky choice, or PR break point. However, the PR breakpoint for the 3/100 reward phase was positively correlated with the slope of the impulsive $(r=.48, p=.020)$ and risky $(r=.50, p=.015)$ choice functions, shown in Figures 6A and 6B, respectively. We also assessed correlations of the four timing measures with choice, but none of these measures correlated with either the mean or slope of the impulsive or risky choice tasks, or with the locomotor activity or PR break point.

\subsection{HPLC results}

The results of the HPLC analysis of the NAC and PFC are presented in Table 1 (see also Supplementary Figure 1 for a representative chromatogram from the NAC). There were significantly greater monoamine concentrations in the NAC, $F(1,21)=68.8, p<.001, \eta_{\mathrm{p}}{ }^{2}=.77$. 
There also were significant differences among the monoamines and their metabolites, $F(6,126)$ $=42.0, p<.001, \eta_{\mathrm{p}}{ }^{2}=.67$, and these differed by brain region, $F(6,126)=33.0, p<.001, \eta_{\mathrm{p}}{ }^{2}=$ .61. Within the NAC, the concentration of DA was higher than all of the other monoamines, whereas within the PFC, 5-HIAA/5-HT turnover was highest followed by DOPAC. Although there were some indications of group differences, there was considerable within-group variance that resulted in no significant differences as a function of rearing condition. As a result, all subsequent analyses focused on individual difference correlations between the neurotransmitter concentrations and performance in the behavioral tasks.

3.6.1. Correlations with locomotor behavior. There were no significant correlations of any of the neurotransmitter or metabolite concentrations and post-rearing locomotor activity. The highest correlations were with PFC DA and DOPAC, but none of the correlations were significant.

3.6.2. Correlations with choice behavior. There were several significant correlations between monoamine/metabolite concentrations and impulsive or risky choice behavior, shown in Figure 7. For impulsive choice behavior, NAC NE concentrations were negatively correlated with the impulsive mean $(r=-.44, p=.036$; Figure $7 \mathrm{~A})$ and positively correlated with the impulsive slope $(r=.45, p=.033$; Figure 7B) and NAC 5-HIAA concentrations were positively correlated with the impulsive slope $(r=.55, p=.007$; Figure 7C). For risky choice behavior, NAC NE $(r=-.44, p=.035 ;$ Figure 7D) and NAC 5-HT $(r=-.43, p=.043$; Figure 7E) concentrations were negatively correlated with the risky mean.

3.6.3. Correlations with timing behavior. An examination of the correlation of the monoamine concentrations with timing measures revealed several significant correlations with end times and the duration of high states of responding, but there were no correlations with start 
or middle times. The most common correlation was with high state duration, and all of the significant correlations with the high state duration are displayed in Figures 8 and 9. There were different correlation patterns in the impulsive choice task, where the two delays to reinforcement were different, compared to the risky choice task where the two delays where the same.

In the impulsive choice task on SS peak trials, PFC 5-HIAA concentration was negatively correlated with the end time of the high state, $r=-.49, p=.017$, and high state duration, $r=-.57$, $p=.005$, and PFC 5-HIAA/5-HT turnover was negatively correlated with the high state duration, $r=-.53, p=.010$ (Figure 8A and 8B). There were no significant correlations with any of the metrics of LL timing behavior.

In the risky choice task, NAC DA was positively correlated with high state duration, $r=$ $.47, p=.023$ on U-L trials (Figure 9D). NAC DOPAC/DA turn-over was negatively correlated with both end times, $r=-.45, p=.030$, and high state duration, $r=-.48, p=.022$, on $\mathrm{C}-\mathrm{S}$ trials, and was negatively correlated with high state duration, $r=-.43, p=.039$, on U-L trials (Figures 9A and 9E). In addition, NAC 5-HT was positively correlated with end times, $r=.52, p=.012$, high state durations, $r=.67, p=.001$, and middle times on $\mathrm{C}-\mathrm{S}$ trials, and on U-L trials was positively correlated with end times, $r=.57, p=.004$, and high state duration, $r=.69, p<.001$, and middle times, $r=.45, p=.033$ (Figures 9B and 9F). Finally, NAC 5-HIAA/5-HT turnover was negatively correlated with end times, $r=-.49, p=.018$, and high state duration, $r=-.55, p=$ .006 , on C-S trials, and was negatively correlated with high state duration, $r=-.46, p=.026$, on U-L trials (Figure 9C and 9G).

3.6.4. Correlations with PR behavior. The only significant correlation involved NAC 5-HIAA/5-HT turnover ratio, which was negatively correlated with PR 1/100 break point, $r=$ $.42, p=.047$, shown in Figure 10 . 


\section{Discussion}

The present experiment examined early-rearing-induced moderation of individual differences in locomotor behavior, impulsive choice, risky choice, peak trial timing, and PR performance, as well as NAC and PFC monoamine concentrations. The IC rats displayed greater locomotor activity, were more impulsive, and had higher PR 1/100 breakpoints than EC rats, but the groups did not differ in risky choice or in their timing behavior. There also were no rearing condition differences in monoamine levels. There were, however, substantial individual differences observed in all tasks, several significant cross-task correlations, as well as substantial correlations between monoamine concentrations in the NAC and PFC and many of the individual difference patterns in the behavioral tasks. Choice behavior across tasks was positively correlated, with the most impulsive rats also displaying the greatest risky choice behavior. Serotonergic and noradrenergic functioning in the NAC significantly correlated with impulsive and risky choice behavior, incentive motivation and the timing of the reward delays within the choice tasks, indicating a clear role for this brain region in contributing to individual differences in choice behavior and related tasks.

\subsection{Individual differences in choice, timing, and motivation}

One of the most striking individual difference patterns was the correlation between impulsive and risky choice (Figure 3). Specifically, the rats that were the most impulsive were also the riskiest, evidenced by a strong positive correlation between the mean of the impulsive and risky choice functions. In addition, there was a positive correlation between the slopes of the two tasks, indicating that the rats that were the most adaptable in one choice task were also the most adaptable in the other choice task. The combination of mean and slope measures suggest that a subset of rats were "impulsive and risky" with strong biases and poor adaptability (Figure 
2), consistent with some previous studies (Baumann \& Odum, 2012; Richards et al., 1999). However, the correlations here were generally stronger than in previous work. Previous studies have typically used impulsive and risky choice procedures with different task demands, which may have diluted the correlation between the two tasks. Therefore, the present findings suggest that previous studies may have underestimated the strength of correlation between impulsive and risky choice (see also Laude et al., 2014 for related evidence). The strong correlation suggests that impulsive and risky choice may rely heavily on shared processes within the domain general valuation system (Peters \& Büchel, 2009).

One relevant factor of the domain general system may be an individual's incentive motivation to earn larger rewards. Here, both impulsive choice and risky choice slopes were positively correlated with PR 3/100 breakpoints, indicating that the rats that were the most adaptable in their choice behavior also showed the greatest willingness to work (Figure 6). It is possible that individual differences in incentive motivation may predict maladaptive impulsive and risky tendencies due to deficits in valuations of different outcomes. This relationship could explain the maladaptive decision making that characterizes schizophrenia (Heerey, Bell-Warren, \& Gold, 2008; Heerey, Robinson, McMahon, \& Gold, 2007), depression (Imhoff, Harris, Weiser, \& Reynolds, 2013), and attention deficit hyperactivity disorder (e.g., Barkley, Edwards, Laneri, Fletcher, \& Metevia, 2001; Drechsler, Rizzo, \& Steinhausen, 2008), which are also associated with motivational deficits (Fervaha et al., 2013; Pizzagalli et al., 2009; Volkow et al., 2010; Ward et al., 2012).

On the other hand, individual differences in timing behavior, measured within the impulsive choice task, did not correlate with impulsive or risky choice. This is surprising given the growing evidence of the importance of timing processes in contributing to individual 
differences in choice behavior (Baumann \& Odum, 2012; Galtress et al., 2012; Heilbronner \& Meck, 2013; Marshall et al., under review; McClure, Podos, \& Richardson, 2014; Smith, Marshall, \& Kirkpatrick, under review). However, Galtress et al. (2012) suggested that measurements of timing within an impulsive choice task may underestimate individual difference correlations with choice behavior as the choices themselves may impact on timing behavior. For example, if a rat rarely chose the LL, then it would have only a limited basis for learning to time the LL duration and this could undermine any timing-choice correlation. Their proposition is consistent with recent research from our laboratory that was conducted in parallel with the present research (Marshall et al., under review), and suggests that future measurements of timing-choice correlations should be conducted with timing measured outside of the choice task.

\subsection{Differential rearing}

Environmental rearing conditions moderated individual differences in locomotor activity, impulsive choice, and PR performance (in the initial 1/100 condition), with IC rats displaying higher rates of activity post-rearing, greater impulsive choice, and higher PR break points (in the PR 1/100 condition) compared to EC rats. Interestingly, there were no correlations of individual differences between the tasks moderated by rearing conditions (e.g., individual differences in post-rearing locomotor activity did not correlate with impulsive choice behavior), suggesting that the rearing environments at least partially overrode the pre-existing individual differences. The present pattern of results is consistent with previous reports of the effects of rearing conditions on reward sensitivity, incentive motivation, and impulsivity (e.g., Kirkpatrick et al., 2013). IC rats have frequently been reported to display greater locomotor activity than EC rats (e.g., Simpson \& Kelly, 2011) and greater response rates when working for rewards (Cain, Green, \& 
Bardo, 2006; Kirkpatrick et al., 2013), which has been interpreted as being due to greater reward sensitivity and incentive motivation in IC rats compared to EC rats (e.g., Beckmann \& Bardo, 2012; Brenes \& Fornaguera, 2008; Fone \& Porkess, 2008; Kirkpatrick et al., 2013; Lapiz et al., 2003).

Previous impulsive choice research has revealed mixed results, with two reports displaying increased impulsive choice in IC rats (Kirkpatrick et al., 2013; Perry et al., 2008) and one study revealing the opposite result (Hellemans et al., 2005). Due to differences in rearing environments and reinforcer type, it is difficult to discern the reason for the different patterns of results in the study by Hellemans et al. (2005). The present study implemented similar rearing conditions and reinforcer types as Kirkpatrick et al. (2013) and Perry et al. (2008), and a similar impulsive choice task as Kirkpatrick et al. (2013) indicating that IC rats do make more impulsive choices when tested under conditions similar to the present study. Further research should elucidate the boundary conditions under which these results hold to understand the different patterns in the literature.

On the other hand, the differential rearing conditions exerted little to no effect on risky choice. To our knowledge, there are no previous studies examining risky choice as a function of rearing environment. One examination of behavior in a rodent version of the Iowa Gambling task found that IC rats were more likely to choose larger rewards even when those choices were disadvantageous (Zeeb et al., 2013). It is therefore possible that the differences in rearing conditions might be more pronounced in situations where the risky option is poorer as is the case in the sub-optimal choice task. Indeed, a recent study in pigeons showed greater attraction to risk in IC-reared pigeons in sub-optimal risky choice behavior (Pattison, Laude, \& Zentall, 2013). 
There also were no effects of rearing environment on any aspects of peak timing. To our knowledge, there have been no previous examinations of timing behavior as a function of different rearing conditions. While further assessments should be conducted before reaching any definitive conclusions, it is possible that the effects of the rearing environment may operate primarily through alterations of reward valuation processes related to impulsive choice, leaving timing processes relatively intact.

\subsection{HPLC analyses}

Despite the rearing-induced behavioral differences, there were no significant differences in NAC or PFC monoamine concentrations, consistent with the idea that differential rearing produces changes in neurotransmitter functionality rather than the corresponding basal levels (Stairs \& Bardo, 2009). There were, however, multiple relationships between monoamine concentrations in the PFC and NAC and behavior in different tasks (Figures 7-10).

With regard to choice behavior, rats with higher NAC NE levels displayed greater selfcontrol and risk aversion, and rats with higher NAC 5-HT concentrations displayed greater risk aversion (Figure 7). This suggests that basal NE and/or 5-HT levels may play a key role in determining biases towards impulsivity versus self-control and risk proneness versus risk aversion. In addition, NAC NE and 5-HIAA concentrations were positively correlated with the impulsive slope (Figure 7), indicating that higher concentrations of NE and 5-HIAA may promote greater flexibility/adaptability, at least in impulsive choice. The present findings are consistent with previous research showing NAC involvement in impulsive and risky choice (Doya, 2008; Peters \& Büchel, 2011; Platt \& Huettel, 2008). An interesting and related finding was the negative correlation between NAC 5-HIAA/5-HT turn-over and progressive ratio breakpoints (Figure 10), indicating that rats with greater 5-HT turn-over showed greater 
elasticity of reward (indexed by earlier break points) in the PR 1/100 task. Elasticity reflects the value of a reward in relation to its cost, which in the PR task is the amount of work required to earn the reward, and therefore closely tied to the motivational value of the reward. This indicates that NAC 5-HT may be playing an important role in incentive motivational processes, which has been previously suggested (Galtress \& Kirkpatrick, 2010; Olausson et al., 2006; Peters \& Büchel, 2011; Robbins \& Everitt, 1996; Zhang, Balmadrid, \& Kelley, 2003). The NAC has also been implicated as a potential source for domain general valuation of rewards (Peters \& Büchel, 2009), which is consistent with the patterns observed in the present study, as NAC monoamine concentrations would be expected to then correlate with aspects of both choice tasks. This suggests that the NAC should be examined more extensively to understand the nature of its involvement in choice behavior, particularly in promoting comorbidities in impulsive and risky choice.

With regard to timing behavior, the PFC was primarily involved in impulsive choice timing behavior (Figure 8), with greater 5-HIAA and 5-HIAA/5-HT concentrations associated with shorter high state durations. On the other hand, the NAC was primarily involved in risky choice timing behavior (Figure 9), with high state durations showing a positive correlation with DA and 5-HT levels, but negative correlations with DOPAC/DA and 5-HIAA/5-HT levels. The pattern of results in the NAC indicate that higher basal DA and 5-HT levels led to longer high state durations, whereas higher DA and 5-HT turnover rates were associated with shorter high state durations. Shorter high state durations are indicative of shaper peaks in timing functions, which are associated with greater precision (less noise) in timing processes (e.g., Gibbon \& Church, 1984). Therefore, it appears that DA and 5-HT were involved in timing precision, with increased precision potentially due to increased functional levels (i.e., turnover) of those 
neurotransmitters. Both DA and 5-HT have previously been associated with interval timing processes (Coull, Cheng, \& Meck, 2011), so the present pattern of results is consistent with and extends upon previous reports.

The dissociation between NAC and PFC involvement in the two tasks may be explained by the involvement of the PFC in interval timing (e.g., Coull et al., 2011) and the importance of timing the differential delays in impulsive choice tasks (cf., Galtress et al., 2012). In contrast, the delays to reward were the same in the risky choice task, and the NAC involvement in timing may have reflected the contribution of single-interval timing processes, consistent with previous research showing later end times in responding in single interval timing following NAC lesions (Galtress \& Kirkpatrick, 2010).

\subsection{Conclusion}

Individual differences in impulsive choice predict the onset and success of treatments of substance abuse (MacKillop \& Kahler, 2009; Perry \& Carroll, 2008; Washio et al., 2011). Additionally, individuals susceptible to risky decision making, such as pathological gamblers, show heightened impulsivity relative to control subjects (Alessi \& Petry, 2003; Dixon, Marley, \& Jacobs, 2003; Reynolds, 2006). Elucidating the psychological and neurobiological source of such differences has become a crucial endeavor (Potenza, 2009; Winstanley, 2011). Furthermore, determining how individual differences are moderated by other factors such as rearing environment may aid in the development of more effective intervention techniques to alleviate maladaptive behaviors. The present results suggest that individual differences in choice behavior may originate from domain general processes such as reward sensitivity and incentive motivation tied to basal NAC monoamine concentrations, but may also be affected by domainspecific processes. Rearing environment may operate primarily on impulsive choice, suggesting 
that future studies should examine domain-specific processes related more specifically to impulsive choice. 


\section{Acknowledgements}

The authors would like to thank Dr. Tiffany Galtress for her assistance with data collection and Dr. Mary Cain for use of the enrichment cage and novel objects. The results were presented at the annual meeting of the Eastern Psychological Association in March, 2013. This research was partially supported by NIMH grant RO1-MH085739 awarded to Kimberly Kirkpatrick and Kansas State University. Aaron Smith is now at the Department of Psychology, University of Kentucky. 


\section{References}

Ainslie, G. (1975). Specious reward: a behavioral theory of impulsiveness and impulse control. Psychological Bulletin 82(4), 463-496.

Alessi, S. M., \& Petry, N. M. (2003). Pathological gambling severity is associated with impulsivity in a delay discounting procedure. Behavioral Processes, 64, 345-354.

Ballard, K., \& Knutson, B. (2009). Dissociable neural representations of future reward magnitude and delay during temporal discounting. Neuroimage, 45, 143-150. doi: 10.1016/j.neuroimage.2008.11.004

Barkley, R. A., Edwards, G., Laneri, M., Fletcher, K., \& Metevia, L. (2001). Executive functioning, temporal discounting, and sense of time in adolescents with attention deficit hyperactivity disorder (ADHD) and oppositional defiant disorder (ODD). Journal of Abnormal Child Psychology, 29(6), 541-556.

Baumann, A. A., \& Odum, A. L. (2012). Impulsivity, risk taking and timing. Behavioural Processes, 90, 408-414.

Beckmann, J. S., \& Bardo, M. T. (2012). Environmental enrichment reduces attribution of incentive salience to a food-associated stimulus. Behavioural Brain Research, 226, 331334.

Bickel, W. K., \& Marsch, L. A. (2001). Toward a behavioral economic understanding of drug dependence: delay discounting processes. Addiction, 96(1), 73-86.

Brenes, J., \& Fornaguera, J. (2008). Effects of environmental enrichment and social isolation on sucrose consumption and preference: associations with depressive-like behavior and ventral striatum dopamine. Neuroscience Letters, 436, 278-282. 
Brenes, J., Rodriguez, O., \& Fornaguera, J. (2008). Differential effect of environmental enrichment and social isolation on depressive-like behavior, spontaneous activity and serotonin and norpinephrine concentration in prefrontal cortex and ventral striatum. Pharmacology, Biochemistry and Behavior, 89, 85-93.

Brunner, D. a. R. H. (1997). Insights into the neurobiology of impulsive behavior from serotonin receptor knockout mice. Annals of the New York Academy of Sciences, 836, 81-105.

Cain, M. E., Green, T. A., \& Bardo, M. T. (2006). Environmental enrichment decreases responding for visual novelty. Behavioural Processes, 73(360-366).

Campbell-Meiklejohn, D., Wakeley, J., Herbert, V., Cook, J., Scollo, P., Ray, M. K., .. . Rogers, R. D. (2011). Serotonin and dopamine play complementary roles in gambling to recover losses. Neuropsychopharmacology, 36, 402-410. doi: 10.1038/npp.2010.170

Cardinal, R. N., \& Howes, N. J. (2005). Effects of lesions of the nucleus accumbens core on choice between small certain rewards and large uncertain rewards in rats. BMC Neuroscience, 6(37), 1-19. doi: 10.1186/1471-2202-6-37

Carroll, M. E., Anker, J. J., \& Perry, J. L. (2009). Modeling risk factors for nicotine and other drug abuse in the preclinical laboratory. Drug and Alcohol Dependence.

Church, R. M., Meck, W. H., \& Gibbon, J. (1994). Application of scalar timing theory to individual trials. Journal of Experimental Psychology: Animal Behavior Processes, 20(2), 135-155.

Cocker, P. J., Dinelle, K., Kornelson, R., Sossi, V., \& Winstanley, C. A. (2012). Irrational choice under uncertainty correlates with lower striatal $\mathrm{D}_{2 / 3}$ receptor binding in rats. The Journal of Neuroscience, 32(44), 15450-15457. 
Coull, J. T., Cheng, R.-K., \& Meck, W. H. (2011). Neuroanatomical and neurochemical substrates of timing. Neuropsychopharmacology, 36, 3-25.

de Wit, H. (2008). Impulsivity as a determinant and consequence of drug use: a review of underlying processes. Addiction Biology, 14, 22-31.

Dixon, M. R., Marley, J., \& Jacobs, E. A. (2003). Delay discounting by pathological gamblers. Journal of Applied Behavior Analysis, 36(4), 449-458.

Doya, K. (2008). Modulators of decision making. Nature Neuroscience, 11(4), 410-416.

Drechsler, R., Rizzo, P., \& Steinhausen, H.-C. (2008). Decision-making on an explicit risktaking task in preadolescents with attention-deficit/hyperactivity disorder. Journal of Neural Transmission, 115(2), 201-209. doi: 10.1007/s00702-007-0814-5

Evenden, J. L. (1999). Varieties of impulsivity. Psychopharmacology, 146, 348-361.

Fervaha, G., Graff-Guerrero, A., Zakzanis, K. K., Foussias, G., Agid, O., \& Remington, G. (2013). Incentive motivation deficits in schizophrenia reflect effort computation impairments during cost-benefit decision-making. Journal of Psychiatric Research, 47(11), 1590-1596. doi: 10.1016/j.jpsychires.2013.08.003

Fone, K. C. F., \& Porkess, M. V. (2008). Behavioural and neurochemical effects of post-weaning social isolation in rodents - relevance to development neuropsychiatric disorders. Neuroscience and Biobehavioral Reviews, 32, 1087-1102.

Galtress, T., Garcia, A., \& Kirkpatrick, K. (2012). Individual differences in impulsive choice and timing in rats. Journal of the Experimental Analysis of Behavior, 98(1), 65-87.

Galtress, T., \& Kirkpatrick, K. (2009). Reward value effects on timing in the peak procedure. Learning and Motivation, 40(2), 109-131. doi: doi:10.1016/j.lmot.2008.05.004 
Galtress, T., \& Kirkpatrick, K. (2010). The role of the nucleus accumbens core in impulsive choice, timing, and reward processing. Behavioral Neuroscience, 124(1), 26-43.

Garcia, A., \& Kirkpatrick, K. (2013). Impulsive choice behavior in four strains of rats: Evaluation of possible models of Attention-Deficit/Hyperactivity Disorder. Behavioural Brain Research, 238, 10-22.

Gibbon, J., \& Church, R. M. (1984). Sources of variance in an information processing theory of timing. In H. L. Roitblat, T. G. Bever \& H. S. Terrace (Eds.), Animal Cognition (pp. $465-$ 488). Hillsdale, NJ: Elrbaum.

Haldane, J. B. S. (1956). The estimation and significance of the logarithm of a ratio of frequencies. Annals of Human Genetics, 20(4), 309-311. doi: 10.1111/j.14691809.1955.tb01285.x

Heerey, E. A., Bell-Warren, K. R., \& Gold, J. M. (2008). Decision-making impairments in the context of intact reward sensitivity in schizophrenia. Biological Psychiatry, 64(1), 62-69. doi: 10.1016/j.biopsych.2008.02.015

Heerey, E. A., Robinson, B. M., McMahon, R. P., \& Gold, J. M. (2007). Delay discounting in schizophrenia. Cognitive Neuropsychiatry, 12(3), 213-221. doi: $10.1080 / 13546800601005900$

Heilbronner, S. R., \& Meck, W. H. (2013). Dissociations between interval timing and intertemporal choice following administration of flouxetine, cocaine, or methamphetamine. Behavioural Processes.

Hellemans, K. G. C., Nobrega, J. N., \& Olmstead, M. C. (2005). Early environmental experience alters baseline and ethanol-induced cognitive impulsivity: relationship to forebrain 5HT1A receptor binding. Behavioural Brain Research, 159 207-220. 
Imhoff, S., Harris, M., Weiser, J., \& Reynolds, B. (2013). Delay discounting by depressed and non-depressed adolescent smokers and non-smokers. Drug and Alcohol Dependence. doi: 10.1016/j.drugalcdep.2013.11.014

Jimura, K., Myerson, J., Hilgard, J., Keighley, J., Braver, T. S., \& Green, L. (2011). Domain independence and stability in young and older adults' discounting of delayed rewards. Behavioural Processes, 87(3), 253-259.

Kahneman, D., \& Tversky, A. (1979). Prospect theory: an analysis of decision under risk. Econometrica, 47(2), 263-291. doi: 10.2307/1914185

Kirby, K. N. (2009). One-year temporal stability of delay-discount rates. Psychonomic Bulletin \& Review, 16(3), 457-462.

Kirkpatrick, K., Marshall, A. T., Clarke, J., \& Cain, M. E. (2013). Environmental rearing effects on impulsivity and reward sensitivity. Behavioral Neuroscience, 127(5), 712-724. doi: $10.1037 / \mathrm{a} 0034124$

Lapiz, M. D. S., Fulford, A., Muchimapura, S., Mason, R., Parker, T., \& Marsden, C. A. (2003). Influence of postweaning social isolation in the rat on brain development, conditioned behavior, and neurotransmission. Neuroscience and Behavioral Physiology, 33, 13-29. doi: $10.1023 / \mathrm{A}: 1021171129766$

Laude, J. R., Beckman, J. S., Daniels, C. W., \& Zentall, T. R. (2014). Impulsivity affects suboptimal gambling-like choice by pigeons. Journal of Experimental Psychology: Animal Learning and Cognition, 40(1), 2-11. doi: 10.1037/xan0000001

MacKillop, J., \& Kahler, C. W. (2009). Delayed reward discounting predicts treatment response for heavy drinkers receiving smoking cessation treatment. Drug and Alcohol Dependence, 104(3), 197-203. doi: 10.1016/j.drugalcdep.2009.04.020 
Marco, R., Miranda, A., Melia, A., MUller, U., Butler, L., Gabriels, I., . . Sonuga-Barke, E. J. S. (2009). Delay and reward choice in ADHD: An experimental test of the role of delay aversion. Neuropsychology, 23(3), 367-380.

Marshall, A. T., \& Kirkpatrick, K. (2013). The effects of the previous outcome on probabilistic choice in rats. Journal of Experimental Psychology: Animal Behavior Processes, 39(1), 24-38. doi: $10.1037 / \mathrm{a} 0030765$

Marshall, A. T., Smith, A. P., \& Kirkpatrick, K. (under review). Mechanisms of impulsive choice: I. Individual differences in timing and reward processing. Journal of the Experimental Analysis of Behavior.

Matusiewicz, A. K., Carter, A. E., Landes, R. D., \& Yi, R. (2013). Statistical equivalence and test-retest reliability of delay and probability discounting using real and hypothetical rewards. Behavioural Processes.

Mazur, J. E. (1987). An adjusting procedure for studying delayed reinforcement. In M. L. Commons, J. E. Mazur, J. A. Nevin \& H. Rachlin (Eds.), Quantitative analyses of behavior. Vol. 5. The effect of delay and of intervening events on reinforcer value (pp. 55-73). Hillsdale, NJ: Erlbaum.

Mazur, J. E. (2001). Hyperbolic value addition and general models of animal choice. Psychological Review 108(1), 96-112.

McClure, J., Podos, J., \& Richardson, H. N. (2014). Isolating the delay component of impulsive choice in adolescent rats. Frontiers in Integrative Neuroscience, 8(3). doi:

10.3389/fnint.2014.00003 
Mobini, S., Body, S., Ho, M. Y., Bradshaw, C. M., Szabadi, E., Deakin, J. F., \& Anderson, I. M. (2002). Effects of lesions of the orbitofrontal cortex on sensitivity to delayed and probabilistic reinforcement. Psychopharmacology, 160(3), 290-298.

Myerson, J., Green, L., Hanson, J. S., Holt, D. D., \& Estle, S. J. (2003). Discounting delayed and probabilistic rewards: Processes and traits. Journal of Economic Psychology, 24, 619635.

Odum, A. L. (2011a). Delay discounting: I'm a k, you're a k. Journal of the Experimental Analysis of Behavior, 96(3), 427-439.

Odum, A. L. (2011b). Delay discounting: Trait variable? Behavioural Processes, 87(1), 1-9.

Odum, A. L., \& Baumann, A. A. L. (2010). Delay discounting: state and trait variable. In G. J. Madden \& W. K. Bickel (Eds.), Impulsivity: The behavioral and neurological science of discounting (pp. 39-65). Washington,DC,: APA Books.

Ohmura, Y., Takahashi, T., Kitamura, N., \& Wehr, P. (2006). Three-month stability of delay and probability discounting measures. Experimental and Clinical Psychopharmacology, 14, 318-328.

Olausson, P., Jentsch, J. D., Tronson, N., Neve, R. L., Nestler, E. J., \& Taylor, J. R. (2006). $\Delta \mathrm{FoSB}$ in the nucleus accumbens regulates food-reinforced instrumental behavior and motivation. The Journal of Neuroscience, 26(36), 9196-9204.

Pattison, K. F., Laude, J. R., \& Zentall, T. R. (2013). Environmental enrichment affects suboptimal, risky, gambling-like choice by pigeons. Animal Cognition, 16, 429-434.

Perry, J. L., \& Carroll, M. E. (2008). The role of impulsive behavior in drug abuse. Psychopharmacology, 200, 1-26. 
Perry, J. L., Stairs, D. J., \& Bardo, M. T. (2008). Impulsive choice and environmental enrichment: Effects of d-amphetamine and methylphenidate. Behavioural Brain Research.

Peters, J., \& Büchel, C. (2009). Overlapping and distinct neural systems code for subjective value during intertemporal and risky decision making. The Journal of Neuroscience, 29, $15727-15734$.

Peters, J., \& Büchel, C. (2011). The neural mechanisms of inter-temporal decision-making: understanding variability. Trends in Cognitive Sciences, 15(5), 227-239.

Pizzagalli, D. A., Holmes, A. J., Dillon, D. G., Goetz, E. L., Birk, J. L., Bogdan, R., . . Fava, M. (2009). Reduced caudate and nucleus accumbens response to rewards in unmedicated subjects with major depressive disorder. American Journal of Psychiatry, 166(6), 702710. doi: 10.1176/appi.ajp.2008.08081201

Platt, M. L., \& Huettel, S. A. (2008). Risky business: The neuroeconomics of decision making under uncertainty. Nature Neuroscience, 11(4), 398-403.

Potenza, M. N. (2009). The importance of animal models of decision making, gambling, and related behaviors: implications for translational research in addiction. Neuropsychopharmacology, 34, 2623-2624. doi: 10.1038/npp.2009.152

Rachlin, H., Raineri, A., \& Cross, D. (1991). Subjective probability and delay. Journal of the Experimental Analysis of Behavior, 55(2), 233-244. doi: 10.1901/jeab.1991.55-233

Rahman, S., \& Bardo, M. T. (2008). Environmental enrichment increases ampheramine-induced glutamate neurotransmission in the nucleus accumbens: a neurochemical study. Brain Research, 1197, 40-46. 
Renner, M. J., \& Rosenzweig, M. R. (1987). Enriched and impoverished environments: effects on the brain and behavior. New York: Springer-Verlag.

Reynolds, B. (2006). A review of delay-discounting research with humans: Relations to drug use and gambling. Behavioural Pharmacology, 17, 651-667.

Richards, J. B., Zhang, L., Mitchell, S. H., \& de Wit, H. (1999). Delay or probability discounting in a model of impulsive behavior: effect of alcohol. Journal of the Experimental Analysis of Behavior, 71, 121-143.

Robbins, T. W., \& Everitt, B. J. (1996). Neurobehavioural mechanisms of reward and motivation. Current Opinion in Neurobiology, 6, 228 - 236.

Roberts, S. (1981). Isolation of an internal clock. Journal of Experimental Psychology: Animal Behavior Processes, 7(3), 242-268.

Simpson, J., \& Kelly, J. P. (2011). The impact of environmental enrichment in laboratory rats behavioural and neurochemical aspects. Behavioural Brain Research, 222, 246-264.

Smith, A. P., Marshall, A. T., \& Kirkpatrick, K. (under review). Mechanisms of impulsive choice: II. Timing interventions to promote self-control. Journal of the Experimental Analysis of Behavior.

Stairs, D. J., \& Bardo, M. T. (2009). Neurobehavioral effects of environmental enrichment and drug abuse vulnerability. Pharmacology, Biochemistry and Behavior, 92, 377-382.

Stopper, C. M., \& Floresco, S. B. (2011). Contributions of the nucleus accumbens and its subregions to different aspects of risk-based decision making. Cognitive and Affective Behavioral Neuroscience, 11, 97-112.

Tatham, T. A., \& Zurn, K. R. (1989). The Med-PC experimental apparatus programming system. Behavior Research Methods, Instruments, and Computers, 21(2), 294-302. 
Volkow, N. D., Wang, G.-J., Newcorn, J. H., Kollins, S. H., Wigal, T. L., Telang, F., .. . Swanson, J. M. (2010). Motivation deficit in ADHD is associated with dysfunction of the dopamine reward pathway. Molecular Psychiatry, 15, 1147-1154. doi: $10.1038 / \mathrm{mp} .2010 .97$

Ward, R. D., Simpson, E. H., Richards, V. L., Deo, G., Taylor, K., Glendinning, J. I., . . . Balsam, P. D. (2012). Dissociation of hedonic reaction to reward and incentive motivation in an animal model of negative symptoms of schizophrenia. Neuropsychopharmacology, 37, 1699-1707. doi: 10.1038/npp.2012.15

Washio, Y., Higgins, S. T., Heil, S. H., McKerchar, T. L., Badger, G. J., Skelly, J. M., \& Dantona, R. L. (2011). Delay discounting is associated with treatment response among cocaine-dependent outpatients. Experimental and Clinical Psychopharmacology, 19(3), 243-248. doi: 10.1037/a0023617

Winstanley, C. A. (2011). Gambling rats: insight into impulsive and addictive behavior. Neuropsychopharmacology, 36(1), 359. doi: 10.1038/npp.2010.136

Winstanley, C. A., Theobald, D. E. H., Dalley, J. W., Cardinal, R. N., \& Robbins, T. W. (2006). Double dissociation between serotonergic and dopaminergic modulation of medial prefrontal and orbitofrontal cortex during a test of impulsive choice. Cerebral Cortex, 16, 106-114.

Wood, D. A., Buse, J. E., Wellman, C. L., \& Rebec, G. V. (2005). Differential environmental exposure alters NMDA but not AMPA receptor subunit expression in nucleus accumbens core and shell. Brain Research, 1042, 176-183. doi: 10.1016/j.brainres.2005.02.029 
Wood, D. A., \& Rebec, G. V. (2009). Environmental enrichment alters neuronal processing in the nucleus accumbens core during appetitive conditioning. Brain Research, 1259, 59-67. doi: 10.1016/j.brainres.2008.12.038

Zeeb, F. D., Wong, A. C., \& Winstanley, C. A. (2013). Differential effects of environmental enrichment, social-housing, and isolation-rearing on a rat gambling task: Dissociations between impulsive action and risky decision-making. Psychopharmacology (Berlin), 225, 381-395. doi: 10.1007/s00213-012-2822-x

Zhang, M., Balmadrid, C., \& Kelley, A. (2003). Nucleus accumbens opiod, GABAergic and dopaminergic modualtion of palatable food motivation: Contrasting effects revealed by a progressive ratio study in the rat. Behavioral Neuroscience, 117(2), 202-211.

Zhong, S., Israel, S., Xue, H., Sham, P. C., Ebstein, R. P., \& Chew, S. H. (2009). A neurochemical approach to valuation sensitivity over gains and losses. Proceedings of the Royal Society B, 276, 4181-4188. doi: 10.1098/rspb.2009.1312

Zhu, J., Apparsundarum, S., Bardo, M. T., \& Dwoskin, L. P. (2005). Environmental enrichment decreases cell surface expression of the dopamine transporter in rat medial prefrontal cortex. Journal of Neurochemistry, 93(1), 434-443. 
Table 1. Mean \pm standard deviation concentration of monoamines in the nucleus accumbens and medial prefrontal cortex, measured by HPLC (in $\mathrm{ng} / \mathrm{mg}$ ), in EC and IC rats.

\begin{tabular}{lcc}
\hline & EC & IC \\
\hline Nucleus Accumbens & $3.29 \pm 0.22$ & $2.80 \pm 0.27$ \\
NE & $2.59 \pm 0.34$ & $2.49 \pm 0.27$ \\
DOPAC & $6.32 \pm 0.98$ & $6.75 \pm 1.02$ \\
DA & $0.50 \pm 0.08$ & $0.52 \pm 0.14$ \\
DOPAC/DA & $2.54 \pm 0.17$ & $2.17 \pm 0.20$ \\
5-HIAA & $1.20 \pm 0.10$ & $1.22 \pm 0.10$ \\
5-HT & $2.27 \pm 0.24$ & $2.16 \pm 0.52$ \\
5-HIAA/5-HT & & \\
& & \\
Prefrontal Cortex & $0.54 \pm 0.04$ & $0.57 \pm 0.04$ \\
NE & $1.24 \pm 0.54$ & $1.54 \pm 0.64$ \\
DOPAC & $0.57 \pm 0.21$ & $0.73 \pm 0.28$ \\
DA & $0.67 \pm 0.34$ & $0.82 \pm 0.37$ \\
DOPAC/DA & $0.78 \pm 0.06$ & $0.87 \pm 0.05$ \\
5-HIAA & $0.23 \pm 0.02$ & $0.23 \pm 0.01$ \\
5-HT & $3.53 \pm 0.38$ & $3.97 \pm 0.29$ \\
5-HIAA/5-HT & & \\
\hline
\end{tabular}




\section{Figure Captions}

Figure 1. Total distance moved (mean \pm standard error of the mean, SEM) in meters $(m)$ as a function of rearing condition during the pre- and post-rearing assessments for EC and IC rats.

Figure 2. A. Mean $( \pm$ SEM) log odds of impulsive (SS) choices as a function of LL magnitude in EC and IC rats. The corresponding percentage SS choices is provided next to each data point. B. Mean impulsive (SS) choices, collapsed across reward magnitude, versus the slope of the impulsive choice function for individual EC and IC rats. A polynomial function is fitted through the distribution for each group. C. Mean $( \pm$ SEM) log odds of risky $(U-L)$ choices as a function of U-L probability for EC and IC rats, with corresponding percentage U-L choices presented next to each data point. D. Mean risky choice versus the slope of the risky choice function for individual EC and IC rats. Polynomial functions are fitted through the distributions.

Figure 3. A. The mean of the impulsive choice versus risky choice functions for individual EC and IC rats. The solid line is the best-fitting regression function through the combined data from the two rearing conditions. B. The slope of the impulsive choice versus risky choice functions for the individual EC and IC rats. The solid line is the best-fitting regression function through the combined data from the two rearing conditions.

Figure 4. Mean $( \pm$ SEM) start $(S)$, middle $(M)$, end $(E)$, and duration $(D)$ times of high states of responding obtained from the low-high-low algorithm for EC and IC rearing conditions for the SS (A) and LL (B) levers in the impulsive choice task and the C-S (C) and U-L (D) levers in the risky choice task.

Figure 5. The effect of reward magnitude or probability on the mean $( \pm$ SEM $)$ breakpoint for EC and IC rats during the PR task.

Figure 6. The PR breakpoint versus impulsive slope (A) or risky slope (B) for individual EC and IC rats. 
Figure 7. NAC NE concentration (ng/mg) versus impulsive mean (A), impulsive slope (B) or risky mean (D), NAC 5-HIAA concentration (ng/mg) versus impulsive slope (C) and NAC 5-HT (ng/mg) versus risky mean $(\mathbf{E})$.

Figure 8. Correlations of PFC 5-HIAA (A), and 5-HIAA/5-HT turnover (B) with high state duration during the impulsive choice task on the SS lever. The high state duration was obtained from the low-high-low algorithm. The solid line is the best-fitting regression function through the combined data from the two rearing conditions.

Figure 9. Correlations of NAC DOPAC/DA turnover (A), NAC 5-HT (B), and NAC 5-HIAA/5HT turnover $(\mathbf{C})$ with high state duration in the risky choice task on the C-S lever, and of NAC DA (D), NAC DOPAC/DA (E), NAC 5-HT (F), and NAC 5-HIAA/5-HT turnover (G) with high state duration in the risky choice task on the U-L lever for individual EC and IC rats. The high state duration was obtained from the low-high-low algorithm. The solid line is the best-fitting regression function through the combined data from the two rearing conditions.

Figure 10. NAC 5-HIAA/5-HT turnover ratio versus PR breakpoint in the 1/100 reward condition for individual EC and IC rats. The solid line is the best-fitting regression function through the combined data from the two rearing conditions.

Supplementary Figure 1. Chromatograms of the sample (NAC), standard ( $1 \mathrm{ng} / \mu \mathrm{l})$ and blank (effluent only) runs. Note that the blank did not mask any of the sample or standard runs. 